\title{
ON THE ZEROS OF SUCCESSIVE DERIVATIVES OF INTEGRAL FUNCTIONS
}

\author{
BY
}

SHEILA SCOTT MACINTYRE

1. The Gontcharoff polynomials

$G_{0}(z)=1 ; G_{n}\left(z ; z_{1}, z_{2}, \cdots, z_{n}\right)=\int_{z_{1}}^{z} d z^{\prime} \int_{z_{2}}^{z^{\prime}} d z^{\prime \prime} \cdots \int_{z_{n}}^{z^{(n-1)}} d z^{(n)} \quad(n \geqq 1)$

have applications to a certain class of interpolation problem (Whittaker $[7])\left({ }^{1}\right)$. In this paper I obtain some formulae connected with these polynomials and use them to improve and extend a theorem due to Levinson [ 3 , 4 ], and to shorten the proof of and extend a theorem due to Schoenberg [6].

LEvinson's THEOREM. If $f(z)$ is an integral function satisfying

$$
\limsup _{r \rightarrow \infty} \frac{\log M(r)}{r}<.7199,
$$

and if $f(z)$ and each of its derivatives have at least one zero in or on the unit circle, then $f(z) \equiv 0$.

The constant .7199 is not the "best possible" but cannot be replaced [5] by a number as great as .7378 .

The "best possible" value of this constant is known as the Whittaker constant $W$. Among new results in this paper, I prove that $W$ cannot be less than .7259.

Schoenberg's Theorem. If $f(z)$ is an integral function satisfying

$$
\limsup _{r \rightarrow \infty} \frac{\log M(r)}{r}<\frac{\pi}{4}
$$

and if $f(z)$ and each of its derivatives have at least one zero in the segment $-1 \leqq x$ $\leqq 1$ of the real axis, then $f(z) \equiv 0$.

The constant $\pi / 4$ is the "best possible" as shown by the example $\cos (\pi z / 4)+\sin (\pi z / 4)$.

I have to thank Mr. M. H. Quenouille and his staff of computers, Statistics Department, Aberdeen University, for performing the calculations arising in $\$ 3$.

Presented to the Society, October 30, 1948; received by the editors August 17, 1948.

(1) Numbers in brackets refer to the references cited at the end of the paper. 
2. Following the notation used by Levinson [3], let

$$
\begin{aligned}
H_{0}(z) & =1 ; H_{n}\left(z_{1}, z_{2}, \cdots, z_{n}\right)=G_{n}\left(0 ; z_{1}, z_{2}, \cdots, z_{n}\right) & & (n \geqq 1), \\
M_{n} & =\max \left|G_{n}\left(z_{0} ; z_{1}, z_{2}, \cdots, z_{n}\right)\right| & & \left(\text { all }\left|z_{r}\right| \leqq 1\right), \\
L_{n} & =\max \left|H_{n}\left(z_{1}, z_{2}, \cdots, z_{n}\right)\right| & & \left(\text { all }\left|z_{r}\right| \leqq 1\right) .
\end{aligned}
$$

We first require two inequalities (2.1) and (2.4) due to Levinson and (for the sake of completeness) give his proof. Since by definition

$$
G_{n}\left(z_{0} ; z_{1}, \cdots, z_{n}\right)=H_{n}\left(z_{1}, z_{2}, \cdots, z_{n}\right)-H_{n}\left(z_{0}, z_{2}, \cdots, z_{n}\right),
$$

therefore, by Taylor's Theorem

$$
\begin{aligned}
G_{n}\left(z_{0} ; z_{1}, z_{2}, \cdots, z_{n}\right)= & \sum_{r=1}^{n} \frac{\left(z_{1}\right)^{r}}{r !} H_{n-r}\left(z_{r+1}, z_{r+2}, \cdots, z_{n}\right) \\
& -\sum_{r=1}^{n} \frac{\left(z_{0}\right)^{r}}{r !} H_{n-r}\left(z_{r+1}, z_{r+2}, \cdots, z_{n}\right) .
\end{aligned}
$$

Hence

$$
\left|G_{n}\left(z_{0} ; z_{1}, \cdots, z_{n}\right)\right| \leqq \sum_{r=1}^{n} \frac{\left|z_{1}^{r}-z_{0}^{r}\right|}{r !} L_{n-r}
$$

and, if we write $2 \alpha=\arg z_{1}-\arg z_{0}$,

$$
M_{n} \leqq \max _{0 \leqq \alpha \leqq \pi / 2}\left\{\sum_{r=1}^{n} \frac{2|\sin r \alpha|}{r !} L_{n-r}\right\} .
$$

By Euler's formula for homogeneous functions,

$$
n G_{n}=\sum_{r=0}^{n} z_{r} \frac{\partial G_{n}}{\partial z_{r}}
$$

and since

$$
\begin{aligned}
& \frac{\partial G_{n}}{\partial z_{0}}=G_{n-1}\left(z_{0} ; z_{2}, \cdots, z_{n}\right), \\
& \frac{\partial G_{n}}{\partial z_{r}}=-G_{r-1}\left(z_{0} ; z_{1}, \cdots, z_{r-1}\right) \times G_{n-r}\left(z_{r} ; z_{r+1}, \cdots, z_{n}\right) \quad(r \geqq 1),
\end{aligned}
$$

we have the inequality

$$
n M_{n} \leqq M_{n-1}+\sum_{r=1}^{n} M_{r-1} M_{n-r}
$$

It is obvious, as Levinson points out, that $L_{1}=1, L_{2}=3 / 2, M_{1}=2$, and hence from $(2.1)$ he obtains $M_{2} \leqq(3 / 2) 3^{1 / 2}<2.5981, M_{3}<3.6379$. By special 
choice of the $z_{r}$ he shows that these values are "accurate" and that in fact $M_{2}=(3 / 2) 3^{1 / 2}$ and $M_{3}>3.6378$. It can also be proved [4] that $L_{3}=2^{-1}\left[2(5)^{1 / 2}+3\right]^{1 / 2}+6^{-1}\left[6(5)^{1 / 2}-2\right]^{1 / 2}<1.9299$, and again, by use of $(2.1)$ he obtains [4] $M_{4}<4.8414$. He then uses (2.4) to find upper bounds for $M_{5}$, $M_{6}, M_{7}, M_{8}, M_{9}$, and (by induction) $M_{n}$. In fact $M_{n} \leqq r^{n+1}(n>1)$ where $r<1.389$. He remarks that this method would presumably yield a better value of $r$ if accurate values of some further members of the sequence $M_{n}$ were worked out before resorting to the use of formula (2.4). However the problem of determining $L_{4}$ or $M_{5}$ exactly is not simple and for higher $L_{n}, M_{n}$, this does not seem a very promising line of approach.

3. It is, however, possible to obtain upper bounds for $L_{4}$, and so on, by using another interation formula involving both sequences $L_{n}$ and $M_{n}$. For Euler's formula gives

$$
n H_{n}=\sum_{r=1}^{n} z_{r} \frac{\partial H_{n}}{\partial z_{r}}
$$

and since

$$
\partial H_{n} / \partial z_{r}=-H_{r-1}\left(z_{1} ; z_{2}, \cdots, z_{r-1}\right) \times G_{n-r}\left(z_{r} ; z_{r+1}, \cdots, z_{n}\right),
$$

we have the inequality

$$
n L_{n} \leqq \sum_{r=1}^{n} L_{r-1} M_{n-r}
$$

In particular, when $n=4,4 L_{4} \leqq L_{0} M_{3}+L_{1} M_{2}+L_{2} M_{1}+L_{3} M_{0}$, yielding $L_{4}$ $<2.7915$, and (2.1) gives $M_{5} \leqq \max _{0} \leqq_{\alpha} \leqq / 2 \phi_{5}(\alpha)$ where

$$
\begin{aligned}
\phi_{5}(\alpha)= & 5.5830|\sin \alpha|+1.9299|\sin 2 \alpha|+(1 / 2)|\sin 3 \alpha| \\
& +(1 / 12)|\sin 4 \alpha|+(1 / 60)|\sin 5 \alpha| .
\end{aligned}
$$

The maximum on this curve lies between $70^{\circ} 27^{\prime}$ and $70^{\circ} 28^{\prime}$ and shows that $M_{5}<6.8223$.

Proceeding in this way by alternate use of (2.1) and (3.1), we find upper bounds for $L_{5}, L_{6}, L_{7}, L_{8}, L_{9}, L_{10} ; M_{6}, M_{7}, M_{8}, M_{9}$, and $M_{10}$ (see appendix). The curves whose maxima have to be determined may be taken as

$$
\begin{aligned}
\phi_{6}(\alpha)= & 7.6112|\sin \alpha|+2.7915|\sin 2 \alpha|+0.6433|\sin 3 \alpha| \\
& +(1 / 8)|\sin 4 \alpha|+(1 / 60)|\sin 5 \alpha|+(1 / 360)|\sin 6 \alpha| \\
& \quad\left(\text { maximum between } 69^{\circ} 31^{\prime} \text { and } 69^{\circ} 32^{\prime}\right), \\
\phi_{7}(\alpha)= & 10.5078|\sin \alpha|+3.8056|\sin 2 \alpha|+0.9305|\sin 3 \alpha| \\
& +0.1609|\sin 4 \alpha|+(1 / 40)|\sin 5 \alpha|+(1 / 360)|\sin 6 \alpha|+2 / 7 ! \\
& \text { (maximum between } \left.69^{\circ} 54^{\prime} \text { and } 69^{\circ} 55\right),
\end{aligned}
$$




$$
\begin{aligned}
\phi_{8}(\alpha)= & 14.4630|\sin \alpha|+5.2539|\sin 2 \alpha|+1.2686|\sin 3 \alpha| \\
& +0.2327|\sin 4 \alpha|+0.0322|\sin 5 \alpha|+(1 / 240)|\sin 6 \alpha| \\
& +2 / 7 !+2 / 8 ! \quad\left(\text { maximum between } 69^{\circ} 49^{\prime} \text { and } 69^{\circ} 51^{\prime}\right), \\
\phi_{9}(\alpha)= & 19.926924|\sin \alpha|+7.2320|\sin 2 \alpha|+1.7513|\sin 3 \alpha| \\
& +0.31714|\sin 4 \alpha|+0.04653|\sin 5 \alpha|+0.00537|\sin 6 \alpha| \\
& +3 / 7 !+2 / 8 !+2 / 9 ! \quad\left(\text { maximum between } 69^{\circ} 49^{\prime} \text { and } 69^{\circ} 51^{\prime}\right), \\
\phi_{10}(\alpha)= & 27.4424|\sin \alpha|+9.9635|\sin 2 \alpha|+2.4105|\sin 6 \alpha| \\
& +0.437825|\sin 4 \alpha|+0.0634267|\sin 5 \alpha|+0.0077542|\sin 6 \alpha| \\
& +3.8598 / 7 !+3 / 8 !+2 / 9 !+2 / 10 !
\end{aligned}
$$

(maximum between $69^{\circ} 49^{\prime}$ and $69^{\circ} 51^{\prime}$ ).

It can be verified by direct computation that

$$
\begin{array}{rlr}
M_{k}<2(1.3775)^{k+1} & (k=1,2,3), \\
M_{k}<(1.3775)^{k+1} & (k=4,5,6,7,8,9,10), \\
L_{k}<(1.3775)^{k} & (k=1,2,3,4), \\
L_{k}<0.7692(1.3775)^{k} & (k=5,6,7,8,9,10) .
\end{array}
$$

From (3.1) we have

$$
\begin{aligned}
n L_{n}< & M_{n-1}+M_{n-2}+1.5 M_{n-3}+1.9299 M_{n-4}+2.7915 M_{n-5} \\
& +\sum_{r=6}^{n-5} L_{r-1} M_{n-r}+4.8414 L_{n-5}+3.6379 L_{n-4}+2.5981 L_{n-3} \\
& +2 L_{n-2}+L_{n-1} .
\end{aligned}
$$

If we assume (3.3) and (3.5) are satisfied also for $11 \leqq k \leqq n-1$, then (3.6) gives, if we write $\gamma=1.3775, \mu=0.7692$,

$$
\begin{aligned}
n L_{n}< & \gamma^{n}+\gamma^{n-1}+1.5 \gamma^{n-2}+1.9299 \gamma^{n-3}+2.7915 \gamma^{n-4} \\
& +\mu\left[(n-10) \gamma^{n}+4.8414 \gamma^{n-5}+3.6379 \gamma^{n-4}+2.5981 \gamma^{n-3}\right. \\
& \left.+2 \gamma^{n-2}+\gamma^{n-1}\right]<n \mu \gamma^{n}-0.0005 \gamma^{n-5} .
\end{aligned}
$$

Hence $L_{n}<\mu \gamma^{n}$.

This proves (3.5) is true for all $k \geqq 11$, by induction.

From (2.1) for $n \geqq 11$,

$$
\begin{aligned}
M_{n} & \leqq \max _{0 \leqq \alpha \leqq \pi / 2}\left\{\sum_{r=1}^{6} \frac{2|\sin r \alpha|}{r !} L_{n-r}\right\}+\sum_{r=7}^{n} \frac{2}{r !} L_{n-r} \\
& <\mu \gamma^{n-7} \max _{0 \leqq \alpha \leqq \pi / 2} \Phi(\alpha)+\sum_{r=7}^{n} \frac{2}{r !} \gamma^{7-r}
\end{aligned}
$$


where

$$
\Phi(\alpha)=\sum_{r=1}^{6} \frac{2|\sin r \alpha|}{r !} \gamma^{7-r},
$$

which has its maximum between $69^{\circ} 49^{\prime}$ and $69^{\circ} 51^{\prime}$, giving

$$
\max _{0 \leqq \alpha \leqq \pi / 2} \Phi(\alpha)<16.8520 .
$$

Hence

$$
\begin{aligned}
M_{n} & <16.8520 \mu \gamma^{n-7}+\gamma^{n-7}\left[\frac{2}{7 !}+\frac{2}{8 !} \frac{1}{\gamma}+\frac{2}{9 !} \frac{1}{\gamma^{2}}+\cdots\right] \\
& <16.8520 \mu \gamma^{n-7}+\gamma^{n-7} \frac{2}{7 !}\left[1+\frac{1}{8 \gamma}+\frac{1}{(8 \gamma)^{2}}+\cdots\right] \\
& =16.8520 \mu \gamma^{n-7}+\frac{2 \gamma^{n-7}}{7 !(1-1 / 8 \gamma)} \\
& <\gamma^{n-7}[12.9626+0.0006] \\
& <\gamma^{n+1} .
\end{aligned}
$$

This proves (3.3) for all $k \geqq 11$, by induction.

Since $G_{n}$ is analytic in the $z_{r}$ it follows that its maximum modulus is assumed when each $z_{r}$ is on the circumference of the unit circle. Thus we have the following theorem.

THEOREM I. If $z_{r}$ is a sequence of points in the unit circle, then

$$
M_{n}=\max \left|G_{n}\left(z_{0} ; z_{1}, z_{2}, \cdots, z_{n}\right)\right|<(1.3775)^{n+1} \quad(n \geqq 4) .
$$

4. Now consider the Gontcharoff polynomials for the case discussed by Schoenberg, namely $G_{n}\left(x ; x_{1}, x_{2}, \cdots, x_{n}\right)$ where

$$
-1 \leqq x_{r} \leqq+1(1 \leqq r \leqq n) .
$$

Consider any one of the $2^{n-r}$ polynomials

$$
\begin{gathered}
G_{n}\left(x ; x_{1}, x_{2}, \cdots, x_{r}, \pm 1, \pm 1, \cdots, \pm 1\right) \quad(1 \leqq r \leqq n), \\
\frac{\partial G_{n}}{\partial x_{r}}=-G_{r-1}\left(x ; x_{1}, x_{2}, \cdots, x_{r-1}\right) \times G_{n-r}\left(x_{r}, \pm 1, \pm 1, \cdots\right) .
\end{gathered}
$$

As $x_{r}$ varies between -1 and +1 , keeping $x_{1}, x_{2}, \cdots, x_{r-1}$ fixed, $\partial G_{n} / \partial x_{r}$ is of constant sign, that is, $G_{n}\left(x ; x_{1}, \cdots, x_{r}, \pm 1, \pm 1, \cdots, \pm 1\right)$ increases or decreases steadily. Hence $\left|G_{n}\left(x ; x_{1}, \cdots, x_{r}, \pm 1, \pm 1, \cdots, \pm 1\right)\right|$ attains its maximum when $x_{r}$ is an end point.

If we take $r=1,2, \cdots, n$, it follows that $\left|G_{n}\left(x ; x_{1}, \cdots, x_{n}\right)\right|\left(-1 \leqq x_{r}\right.$ 
$\leqq 1)$ attains its maximum for any given value of $x(-1 \leqq x \leqq 1)$ when $x_{r}= \pm 1$ $(1 \leqq r \leqq n)$.

So, in order to find an upper bound for $\left|G_{n}\left(x ; x_{1}, x_{2}, \cdots ; x_{n}\right)\right|\left(-1 \leqq x_{r}\right.$ $\leqq 1)$, it is sufficient to consider the $2^{n}$ polynomials $\left|G_{n}(x ; \pm 1, \pm 1, \cdots, \pm 1)\right|$ $(-1 \leqq x \leqq 1)$.

Clearly if $0 \leqq x \leqq 1$ and $x_{r}= \pm 1$,

$$
\begin{aligned}
\left|G_{n}\left(x ;+1, x_{2}, \cdots, x_{n}\right)\right| & =\left|G_{n}\left(-x ;-1,-x_{2}, \cdots,-x_{n}\right)\right| \\
& \leqq\left|G_{n}\left(0 ;-1,-x_{2}, \cdots,-x_{n}\right)\right| \\
& \leqq\left|G_{n}\left(x ;-1,-x_{2}, \cdots,-x_{n}\right)\right| .
\end{aligned}
$$

I shall prove that if $0 \leqq x \leqq 1$ and $x_{r}= \pm 1(1 \leqq r \leqq n)$ for all $n$,

$$
\left|G_{n}\left(x ; x_{1}, x_{2}, \cdots, x_{n}\right)\right| \leqq 2\left(\frac{4}{\pi}\right)^{n-1} \sin \frac{\pi}{4}(x+1) .
$$

By (4.2), it is sufficient to prove (4.3) for the case $x_{1}=-1$, that is, it is sufficient to prove

$$
\left|G_{n}\left(x ;-1,+1, x_{3}, \cdots, x_{n}\right)\right| \leqq 2\left(\frac{4}{\pi}\right)^{n-1} \sin \frac{\pi}{4}(x+1)
$$

and

$$
\left|G_{n}\left(x ;-1,-1, x_{3}, \cdots, x_{n}\right)\right| \leqq 2\left(\frac{4}{\pi}\right)^{n-1} \sin \frac{\pi}{4}(x+1) .
$$

Proof of (4.4).

$$
\begin{aligned}
\left|G_{n+1}\left(x ;-1,+1, x_{3}, \cdots, x_{n+1}\right)\right| & =\int_{-1}^{x}\left|G_{n}\left(x^{\prime} ;+1, x_{3}, \cdots, x_{n+1}\right)\right| d x^{\prime} \\
& =I_{1}+I_{2},
\end{aligned}
$$

where

$$
\begin{aligned}
& I_{1}=\int_{-1}^{0}\left|G_{n}\left(x^{\prime} ;+1, x_{3}, \cdots, x_{n+1}\right)\right| d x^{\prime}, \\
& I_{2}=\int_{0}^{x}\left|G_{n}\left(x^{\prime} ;+1, x_{3}, \cdots, x_{n+1}\right)\right| d x^{\prime} .
\end{aligned}
$$

If we use (4.1),

$$
I_{1}=\int_{-1}^{0}\left|G_{n}\left(-x^{\prime} ;-1,-x_{3}, \cdots,-x_{n+1}\right)\right| d x^{\prime} .
$$

If we substitute $x=-x^{\prime}$, 


$$
I_{1}=\int_{0}^{1}\left|G_{n}\left(x ;-1,-x_{3}, \cdots,-x_{n+1}\right)\right| d x .
$$

Now if we assume that (4.3) is true if $n$ is replaced by any number $m \leqq n$,

$$
\begin{aligned}
I_{1} & \leqq 2\left(\frac{4}{\pi}\right)^{n-1} \int_{0}^{1} \sin \frac{\pi}{4}(x+1) d x=2^{1 / 2}\left(\frac{4}{\pi}\right)^{n}, \\
I_{2} & =\int_{0}^{x} d x^{\prime} \int_{x^{\prime}}^{1}\left|G_{n-1}\left(x^{\prime \prime} ; x_{3}, \cdots, x_{n+1}\right)\right| d x^{\prime \prime} \\
& \leqq 2\left(\frac{4}{\pi}\right)^{n-2} \int_{0}^{x} d x^{\prime} \int_{x^{\prime}}^{1} \sin \frac{\pi}{4}\left(x^{\prime \prime}+1\right) d x^{\prime \prime} \\
& =2\left(\frac{4}{\pi}\right)^{n} \sin \frac{\pi}{4}(x+1)-2^{1 / 2}\left(\frac{4}{\pi}\right)^{n} .
\end{aligned}
$$

Therefore $I_{1}+I_{2} \leqq 2(4 / \pi)^{n} \sin (\pi / 4)(x+1)$.

But (4.4) is true when $n=0,1$.

Hence (4.4) is true for all $n$ by induction.

Proof of (4.5).

$$
\begin{aligned}
G_{n+1}\left(x ;-1,-1, x_{3}, \cdots, x_{n}\right) & =\int_{-1}^{x}\left|G_{n}\left(x^{\prime} ;-1, x_{3}, \cdots, x_{n}\right)\right| d x^{\prime} \\
& =I_{3}+I_{4}
\end{aligned}
$$

where

$$
\begin{aligned}
& I_{3}=\int_{-1}^{0}\left|G_{n}\left(x^{\prime} ;-1, x_{3}, \cdots, x_{n}\right)\right| d x^{\prime} \\
& I_{4}=\int_{0}^{x}\left|G_{n}\left(x^{\prime} ;-1, x_{3}, \cdots, x_{n}\right)\right| d x^{\prime} .
\end{aligned}
$$

If we use (4.1),

$$
I_{3}=\int_{-1}^{0}\left|G_{n}\left(-x^{\prime} ;+1,-x_{3}, \cdots,-x_{n}\right)\right| d x^{\prime} .
$$

If we substitute $x=-x^{\prime}$,

$$
\begin{aligned}
I_{3} & =\int_{0}^{1}\left|G_{n}\left(x ;+1,-x_{3}, \cdots,-x_{n}\right)\right| d x \\
& =\int_{0}^{1} \int_{x}^{1}\left|G_{n-1}\left(x^{\prime} ;-x_{3}, \cdots,-x_{n}\right)\right| d x^{\prime} .
\end{aligned}
$$

Hence, if we assume (4.3) is true if $n$ is replaced by any number $m \leqq n$, 


$$
\begin{aligned}
I_{3} & \leqq 2\left(\frac{4}{\pi}\right)^{n-2} \int_{0}^{1} d x \int_{x}^{1} \sin \frac{\pi}{4}\left(x^{\prime}+1\right) d x^{\prime} \\
& =\left(\frac{4}{\pi}\right)^{n}\left(2-2^{1 / 2}\right) . \\
I_{4} & \leqq 2\left(\frac{4}{\pi}\right)^{n-1} \int_{0}^{x} \sin \frac{\pi}{4}\left(x^{\prime}+1\right) d x^{\prime} \\
& =\left(\frac{4}{\pi}\right)^{n}\left(-2 \cos \frac{\pi}{4}(x+1)+2^{1 / 2}\right) .
\end{aligned}
$$

Now $1-\cos (\pi / 4)(x+1) \leqq \sin (\pi / 4)(x+1), \quad 0 \leqq x \leqq 1$. Hence $I_{3}+I_{4}$ $\leqq 2(4 / \pi)^{n} \sin (\pi / 4)(x+1)$.

But (4.5) is true when $n=0,1$. Hence (4.5) is true for all $n$ by induction.

Since (4.4) and (4.5) are true, we have proved (4.3). It follows by substituting $-x$ for $x$, that for $-1 \leqq x \leqq 0$ and $-1 \leqq x_{r} \leqq 1(1 \leqq r \leqq n)$,

$$
\left|G_{n}\left(x ; x_{1}, x_{2}, \cdots, x_{n}\right)\right| \leqq 2\left(\frac{4}{\pi}\right)^{n-1} \cos \frac{\pi}{4}(x+1),
$$

and we have the following theorem.

THEOREM II. If $z_{r}$ is a sequence of points on the real axis, satisfying $-1 \leqq z_{r} \leqq 1$, then

$$
\left|G_{n}\left(z_{0} ; z_{1}, z_{2}, \cdots, z_{n}\right)\right| \leqq 2(4 / \pi)^{n-1} .
$$

5. I shall now discuss extensions of Theorems I and II in which some of the points of the sequence $z_{r}$ lie outside the unit circle, and the segment $-1 \leqq x \leqq 1$ respectively.

Let $z_{r}=x_{r}+y_{r}$, where both $x_{r}$ and $y_{r}$ may be complex, then since $G_{n}\left(z_{0} ; z_{1}, \cdots, z_{n}\right)$ is a polynomial in each $z_{r}(0 \leqq r \leqq n)$, we may apply Taylor's series and write

$$
G_{n}\left(z_{0} ; z_{1}, \cdots, z_{n}\right)=\exp \left(\sum_{r=0}^{n} y_{r} \frac{\partial}{\partial x_{r}}\right) G_{n}\left(x_{0} ; x_{1}, \cdots, x_{n}\right) .
$$

Now, writing $G_{n}\left(x_{0} ; x_{1}, x_{2}, \cdots, x_{n}\right)=G_{n}$, using (2.2) and (2.3), we note that $\partial G_{n} / \partial x_{r}(0 \leqq r \leqq n)$ is either one multiple integral or the product of two such integrals, in each case the total multiplicity being $n-1$. Similarly $\partial^{k} G_{n} / \partial x_{r} \partial x_{s} \cdots \partial x_{t}$, where $r, s, \cdots, t$ may all take any values between 0 and $n$ inclusive, is either zero (for example, $\partial^{2} G_{n} / \partial x_{0} \partial x_{1}$ ) or the product of not more than $k+1$ multiple integrals, the total multiplicity being $n-k$.

Now suppose that positive constants $A, \gamma$ can be found such that

$$
\left|G_{n}\right|<A \gamma^{n+1}
$$

provided that the sequence $\left\{x_{r}\right\}$ belongs to a given set of points $S$ which in- 
cludes $z=0$. Such a set exists by Theorem I.

Setting $n=0$, we see that $A \gamma>1$. Hence

$$
\left|\frac{\partial^{k} G_{n}}{\partial x_{r} \partial x_{s} \cdots \partial x_{t}}\right|<A^{k+1} \gamma^{n+1}
$$

Suppose also that the values of $y_{r}$ are restricted in such a way that

$$
\sum_{r=1}^{n}\left|y_{r}\right| \leqq n h
$$

for certain values of $n$. Then (5.1) gives, for these values of $n$,

$$
\begin{aligned}
\left|G_{n}\left(z_{0} ; z_{1}, z_{2}, \cdots, z_{n}\right)\right| & <\sum_{k=0}^{\infty} \frac{\left(\left|y_{0}\right|+n h\right)^{k}}{k !} A^{k+1} \gamma^{n+1} \\
& =A \gamma^{n+1} \exp \left\{A\left(\left|y_{0}\right|+n h\right)\right\} .
\end{aligned}
$$

If the sequence $\left\{z_{r}\right\}$ is such that all its limit points belong to $S$, then (5.3) is satisfied for arbitrarily small $h$ and sufficiently large $n$, and (5.4) gives

$$
\left|G_{n}\left(z_{0} ; z_{1}, \cdots, z_{n}\right)\right|<A e^{A\left|y_{0}\right|}(\gamma+\epsilon)^{n+1}, \quad n \geqq n_{0}(\epsilon),
$$

and hence for all $z$ in any given finite domain, and all $n$,

$$
\left|G_{n}\left(z ; z_{1}, z_{2}, \cdots, z_{n}\right)\right|<A^{\prime}(\gamma+\epsilon)^{n+1} \text {. }
$$

6. Suppose now that $f(z)=\sum_{n=0}^{\infty} a_{n} z^{n}$ is an integral function satisfying

$$
\limsup _{r \rightarrow \infty} \frac{\log M(r)}{r}=\sigma<\frac{1}{r},
$$

it follows that for any $\tau>\sigma$, and sufficiently large $n$

$$
n !\left|a_{n}\right|<\tau^{n} \text {. }
$$

Then, if $f\left(z_{1}\right)=0, f^{(n-1)}\left(z_{n}\right)=0$, clearly

$$
f(z)=\int_{z_{1}}^{z} d z^{\prime} \int_{z_{2}}^{z^{\prime}} d z^{\prime \prime} \cdots \int_{z_{n}}^{z^{(n-1)}} f^{(n)}(z) d z,
$$

or, following Levinson $[3, \S 1]$, if we replace $f^{n}(z)$ by its power series, we obtain

$$
\begin{aligned}
f(z) & =\sum_{k=0}^{\infty}(n+k) ! \frac{a_{n+k}}{k !} \int_{z_{1}}^{z} d z^{\prime} \int_{z_{2}}^{z^{\prime}} d z^{\prime \prime} \cdots \int_{z_{n}}^{z^{(n-1)}} z^{k} d z \\
& =\sum_{k=0}^{\infty}(n+k) ! a_{n+k} G_{n+k}\left(z ; z_{1}, z_{2}, \cdots, z_{n}, 0,0, \cdots, 0\right) .
\end{aligned}
$$

Now since the sequence $\left\{z_{n}\right\}$ is such that all its limit points belong to $S$, then for large $n$ and for all $z$ in any finite domain we have by (5.6) and (6.1) 


$$
|f(z)|<\sum_{k=0}^{\infty} \tau^{n+k} A^{\prime}(\gamma+\epsilon)^{n+k+1}=\frac{A^{\prime}(\gamma+\epsilon)^{n+1} \tau^{n}}{1-\tau(\gamma+\epsilon)}
$$

provided $\tau<1 /(\gamma+\epsilon)$. But letting $n \rightarrow \infty$ in (6.2) we have $f(z) \equiv 0$.

In the particular case in which $S$ is the unit circle, Theorem I shows that (5.2) is satisfied with $\gamma=1.3775<1 / .7259$ for all values of $n$, so we now have the following theorem.

THEOREM III. If $f(z)$ is an integral function satisfying

$$
\limsup _{r \rightarrow \infty} \frac{\log M(r)}{r}<.7259,
$$

and if $f\left(z_{1}\right)=0, f^{(n-1)}\left(z_{n}\right)=0(n \geqq 2)$, the sequence $\left\{z_{r}\right\}$ having all its limit points in the unit circle, then $f(z) \equiv 0$.

In the particular case in which $S$ is the segment $0 \leqq x \leqq 1$, Theorem II shows that (5.2) is satisfied for all $n$ with $\gamma=4 / \pi$ and we have the following extension of Schoenberg's theorem.

THEOREM IV. If $f(z)$ is an integral function satisfying

$$
\limsup _{r \rightarrow \infty} \frac{\log M(r)}{r}<\frac{\pi}{4}
$$

and if $f\left(z_{1}\right)=0, f^{(n-1)}\left(z_{n}\right)=0(n \geqq 2)$, the sequence $\left\{z_{r}\right\}$ having all its limit points on the segment $-1 \leqq x \leqq 1$ of the real axis, then $f(z) \equiv 0$.

This result has been stated by Kamenetsky [2, Theorem VIII] but I have been unable to find a published proof. It seems unlikely from the context that his method has anything in common with the one which I have used here.

7. A further theorem follows as a consequence of inequalities (5.2) and (5.6) for the case in which the limit points of the sequence of zeros lie inside the locus of points distant $h$ from the segment $-1 \leqq x \leqq 1$ of the real axis. We shall call the domain enclosed by this curve $H$. In this case, if we restrict the sequence $\left\{x_{r}\right\}$ to the segment $-1 \leqq x \leqq 1$ (all $r$ ) and $z_{r}=x_{r}+y_{r}$ (all $r \geqq 1$ ) where $\left|y_{r}\right| \leqq h(r \geqq 1),(5.2)$ is satisfied with $A=\pi^{2} / 8, \gamma=4 / \pi$, by Theorem II, and (5.3) is satisfied for all $n$ since $\left|y_{r}\right| \leqq h(r \geqq 1)$. Hence (5.4) is satisfied for all $n$ with these values of the constants, that is,

$$
\left|G_{n}\left(z_{0} ; z_{1}, z_{2}, \cdots, z_{n}\right)\right| \leqq \frac{\pi^{2}}{8}\left(\frac{4}{\pi}\right)^{n+1} \exp \left\{\frac{\pi^{2}}{8}\left(\left|y_{0}\right|+n h\right)\right\}<\bar{A} \bar{\gamma}^{n+1}
$$

with $\bar{\gamma}=(4 / \pi) \exp \left(\pi^{2} h / 8\right)$. By a second application of formulae (5.2) and (5.6), we see that, provided all the limit points of the sequence $\left\{z_{r}\right\}$ lie within $H$, (5.6) holds with $\gamma=(4 / \pi)$ exp $\left(\pi^{2} h / 8\right)$, and we have the following theorem. 
THEOREM V. If $f(z)$ is an integral function satisfying

$$
\limsup _{r \rightarrow \infty} \frac{\log M(r)}{r}<\frac{\pi}{4} \exp \left(-\frac{\pi^{2} h}{8}\right)
$$

and if $f\left(z_{1}\right)=0, f^{(n-1)}\left(z_{n}\right)=0(n \geqq 2)$, where the sequence $\left\{z_{r}\right\}$ has all its limit points in $H$, then $f(z) \equiv 0$.

It is to be noted that the constant $(\pi / 4) \exp \left(-\pi^{2} h / 8\right)$ is "better" (that is, greater) than that obtained from the circle circumscribed to $H$, namely, $.7259 / 1+h$ (which is obtained from Theorem III by the transformation $\zeta=(1+h) z)$ only for small values of $h$. It is "better" when $h \leqq 0.23$ but not when $h=0.24$.

\begin{tabular}{rcccc}
\multicolumn{5}{c}{ APPENDIX } \\
$n$ & $M_{n-1}$ & $L_{n}$ & $L_{n} /(1.3775)^{n}$ & $(1.3775)^{n}$ \\
1 & 1 & 1 & 0.7260 & 1.3775 \\
2 & 2 & 1.5 & 0.7905 & 1.8975 \\
3 & 2.5981 & 1.9299 & 0.7384 & 2.6138 \\
4 & 3.6379 & 2.7915 & 0.7753 & 3.6005 \\
5 & 4.8414 & 3.8056 & 0.7673 & 4.9597 \\
6 & 6.8223 & 5.2539 & 0.7690 & 6.8320 \\
7 & 9.3973 & 7.2315 & 0.7685 & 9.4111 \\
8 & 12.9512 & 9.9635 & 0.7686 & 12.9638 \\
9 & 17.8413 & 13.7212 & 0.7684 & 17.8577 \\
10 & 24.5754 & 18.8998 & 0.7683 & 24.5989 \\
11 & 33.8472 & & & 33.8850
\end{tabular}

\section{REFERENCES}

1. I. M. Kamenetsky, Sur l'interpolation au moyen des dérivées et sur les procédés d'interpolation correspondants I, C. R. Acad. Sci. URSS vol. 25 (1939) pp. 356-358.

2. - Sur l'interpolation au moyen des dérivées et sur les procédés d'interpolation correspondants II, C. R. Acad. Sci. URSS vol. 26 (1940) pp. 217-219.

3. N. Levinson, The Gontcharoff polynomials, Duke Math. J. vol. 11 (1944) pp. 729-733.

4. - Correction to "The Gontcharoff polynomials," Duke Math. J. vol. 12 (1944) p. 335.

5. Sheila Scott Macintyre, An upper bound for the Whittaker constant, J. London Math. Soc. vol. 22 (1947) pp. 305-311.

6. I. J. Schoenberg, On the zeros of successive derivatives of integral functions, Trans. Amer. Math. Soc. vol. 40 (1936) pp. 12-23.

7. J. M. Whittaker, Interpolatory function theory, Cambridge, 1935.

THE University,

AberdeEn, SCOTland 